\title{
La revolución de terciopelo y el movimiento intelectual checoslovaco
}

\author{
Marina Casanova*
}

\begin{abstract}
RESUMEN
ABSTRACT

Se conoce como "Revolución de

The revolution that took place in terciopelo" los acontecimientos que tuvieron lugar en Checoslovaquia en otoño de 1989 y que dieron lugar a la caída del régimen comunista. Aunque calificada de terciopelo por la ausencia de violencia, fue aún más significativo

el medio empleado por la sociedad checa para lograrlo: la palabra.

Por ello hemos analizado, cómo y por qué, algunos intelectuales tales como, Havel, Kundera, Klima, Vaculik, Hrabal y Skvorecky, formaron parte del grupo de disidentes que hicieron posible el cambio.

$\begin{array}{cl}\text { PALABRAS CLAVE } & \text { KEYWORDS } \\ \text { Checoslovaquia. Revolución. Caida } & \text { Czechoslovakia. Revolution. } \\ \text { comunismo. Intelectuales. } & \text { Communist Breakdown. Intellectuals. }\end{array}$
Czechoslovakia in 1989, is known as the "Velvet revolution" for the lack of violence involved to attain democracy. But more significant was the way used by czech society to get it: the word.

Adopting this viewpoint we have analysed, how and why, several intellectuals such as, Havel, Kundera, Klima, Vaculik, Hrabal and Škvorecký, became dissidents, and their contribution to the breakdown of the communist regime.

KEY WORDS

Communist Breakdown. Intellectuals.
\end{abstract}

A partir de 1989, y en un breve espacio de tiempo, terminó la guerra fria, los régimenes autoritarios de los países del este se desplomaron y el imperio soviético se desintegró. Todos estos acontecimientos están ínti-

* UNED. 
mamente conectados, pero su origen, el modo en que se relacionaron unos con otros, cuáles eran predecibles y cuáles fueron verdaderamente inesperados, son tema de controversia de los historiadores. Aunque los archivos de los países del este durante los años 1989-1992 son sólo accesibles parcialmente, algunos protagonistas de los acontecimientos ya han publicado memorias sacando a la luz informaciones que, de otro modo. no hubiera sido posible conocer. Esto no quiere decir que el historiador haya alcanzado la verdadera versión de los hechos, pero el grado de conocimiento que se tiene en la actualidad va siendo cada vez más preciso, aunque se trate de sucesos históricos que aún no han concluido ${ }^{1}$.

Se conoce como “Revolución de terciopelo» los acontecimientos que tuvieron lugar en Checoslovaquia durante los meses de noviembre y diciembre de 1989, que acabaron con el régimen comunista y abrieron el camino a la democracia. Los observadores occidentales la calificaron de "terciopelo", queriendo destacar con ello la ausencia de métodos violentos para lograrlo. Pero fue aún más significativo que la palabra fuese el medio empleado por el pueblo checoslovaco para alcanzar sus objetivos, y por ello, más que «Revolución de terciopelo», convendria llamarla «Revolución de la palabra», aunque seguiremos utilizando aquel término que ya ha sido aceptado por los historiadores.

La variable cíclica en la Historia es un factor frecuentemente destacado cuando se realizan estudios sobre su evolución. En el caso de Checosiovaquia la variable cíclica no sólo aparece de un modo regular cada veinte años, sino que, con mayor precisión, los cambios más significativos de su evolución histórica han tenido lugar sorprendentemente en los años terminados en ocho ". En octubre de 1918 se creó la República checoslovaca. En septiembre de 1938, como consecuencia de los Acuerdos de Munich, el Reich alemán se anexionó los Países checos y se creó un Estado eslovaco pro nazi. Al término de la ll Guerra mundial, en febrero de 1948 tuvo lugar un golpe comunista que destruyó las estructuras democráticas de la jóven República. Durante los veinte años siguientes, Checoslovaquia quedó integrada en el bloque de países comunistas controlados por la Unión Soviética, pero en 1968 surgieron voces de disconformidad que dieron lugar a la "Primavera de Praga". La posterior "normalización" terminó con el "socialismo con rostro humano». En 1988 todos los paises del Este

DAWISHA. K : PABHOI. B.: The consolidation of democracy in East Central Europe Cambridge. Cambridge University Press, 1997, pág. 3.

Skalnik LI.fF. Carol: The Czech and Slovak Republics. Oxford. West View Press. 1997. pág. 44 
empezaron a vivir un momento de cambio, y en Checoslovaquia, siguiendo el rítmo cíclico, como un presagio más soñado que real del número terminado en ocho, se iniciaron campañas de manifestaciones que culminaron en la «Revolución de terciopelo». Y si alguien señala a los checos que los presagios numéricos esta vez no se cumplieron, irónicamente responden que 89 al revés y boca abajo es 68 , precisamente el año en que se vio truncada la "Primavera de Praga».

La "Revolución de terciopelo» tiene forzosamente que enmarcarse junto a los cambios políticos de los países vecinos. Si bien en cada uno de ellos el camino hacia la democracia se desarrolló de diferentes maneras, existen unos antecedentes que afectaron a todos y que, básicamente, se resumen en las diferentes tentativas llevadas a cabo para reformar o trasformar el régimen comunista que les fue impuesto al término de la II Guerra mundial.

El primer intento, o más aún, la primera revolución que tuvo lugar para instaurar la democracia, fue la Revolución húngara de 1956, alentada por las reformas emprendidas por Imre Nagy, y que fue violenta. mente reprimida por el ejército soviético. En Polonia, los intentos de cambio fueron reprimidos en 1956 por Gomulka, que supo convencer a Moscú para que no interviniese militarmente, siendo el propio Partido comunista polaco el que en adelante controlaría las aspiraciones populares de cambio.

En 1968 Alexandre Dubček inició en Checosiovaquia una serie de cambios, dirigidos por el propio Partido, para reformar el sistema político $y$, de este modo, no entrar en conflicto con las autoridades soviéticas. Sin embargo, la supresión de la censura y del control policial difícilmente podían ser bien vistas por la Unión Soviética. Uno de los ideólogos de la "Primavera de Praga", Zdeněk Mlynár, que asistió a la firma de los Acuerdos de Moscú que siginificaron el fin del "socialismo con rostro humano", afirmó que para Brejnev la imágen del socialismo en Occidente le traía sin cuidado y que, lo único que le interesaba era la seguridad de las fronteras de Checoslovaquia, que también lo eran de la Unión Soviética ${ }^{3}$

En todos estos paises los intentos reformistas desaparecieron con la "normalización". Aunque este término puede tener diferentes significados en función de las circunstancias históricas y de quienes lo utilizaron, por lo que se refiere al período aquí señalado, se entiende como el

RupNik, Jacques: L'autre Europe. Paris. Odile Jacob, 1993, pág. 344. 
restablecimiento del control soviético y el dominio y dirigismo del Partido comunista ${ }^{4}$.

La «normalización» llevada a cabo por Husák en Checosiovaquia fue mucho más represiva que la realizada en los países vecinos. Husák, que fue una de las víctimas de las purgas de Stalin, y que apoyó inicialmente las reformas de Dubcek, hizo pensar que llevaria a cabo una «normalización" al estilo de la realizada por Janos Kadar en Hungría, de cooperación con los intelectuales y alejando a la vieja guardia estaliniana. Sin embargo, una vez instalado en el poder, emprendió una total erradicación de las reformas, llamó a los estalinistas más ultras y, en vez de atraerse a los intelectuales, realizó una política de estricto control sobre los ciudadanos y sobre las instituciones. Como decían en Praga: «Hemos sufrido por Husák y bajo Husák».

Los reformadores del Partido comunista comprendieron que cualquier intento de cambio dentro del Partido sería automáticamente abortado por Moscú. Pero la represión emprendida por Husák tuvo un resultado contrario al esperado, y dio lugar a que los llamados "disidentes" buscaran nuevos caminos, dentro de la legalidad, para salir de un régimen que les había sido impuesto por la fuerza. En enero de 1977 se creó la Carta 77 que reunió a un grupo heterogéneo de personas que reclamaron la aplicación de los derechos humanos en Checoslovaquia y que el propio gobierno había ratificado en el Acta final de los Acuerdos de Helsinki. Tomó el nombre de Carta, en referencia a la Carta Magna inglesa otorgada por Juan sin Tierra en 1215, y a la Carta de las Naciones Unidas ${ }^{5}$. Aunque dentro de un círculo muy reducido de intelectuales y escritores, la Carta 77 se convirtió en un símbolo de esperanza y un foco de inconformismo, pero no en una amenaza política la régimen ${ }^{6}$. No obstante, las autoridades comunistas la consideraron como una declaración de guerra. Muchos de los firmantes fueron detenidos, interrogados, sus casas registradas. Pero cuando descubrieron que sólo poseian libros e ideas, les expulsaron de sus trabajos, les retiraron sus pasaportes, sus carnets de conducir. Fue el inicio de la lucha entre los disidentes, que defendian la justicia de sus reivindicaciones, y el sistema, que imponía su superioridad y su fuer$\mathrm{za}^{7}$. A pesar de que los firmantes tuvieron escasos contactos con la po-

4 KIERAN, WILLIAMS: The Prague Spring and its aftermath. Cambridge. Cambridge University Press. 1997, pág. 40.

Duplan, Ch., y GiRet, V.: La vie en rouge. 1969-1989. París. Seuil, 1994, pág. 170.

HAvEl, Václav: Interrogatoire à distance. Eds. de l'Aube. 1989, pág.150-155.

KıimA, Ivan: My golden Trades. London. Pinguin Books. 1990, pág. 104. 
blación que les consideraba como temerarios idealistas ${ }^{8}$, los acontecimientos de 1989 demostraron que la Carta contribuyó a minar el apoyo de la población al Partido, y que sus responsables tuvieron un papel determinante en la creación en 1989 del Foro Civico, organización que encauzaria la «Revolución de terciopelo». y que formaría el primer gobierno no comunista.

El 28 de octubre de 1939 había sido ejecutado en Praga por los nazis el estudiante checo Jan Opletal ${ }^{9}$. El 17 de noviembre de 1989, para conmemorar el 50 aniversario de su muerte, las autoridades habían autorizado una manifestación silenciosa con el mismo recorrido que el del día de su entierro. Quince mil estudiantes llevando velas encendidas salieron del Instituto de Patología en dirección a la tumba del poeta Mácha en el cementerio de vysehrad. Pero otro cortejo más numeroso se unió a los estudiantes, y continuó hasta el centro de la ciudad donde se encuentra la estatua de Opletal. La manifestación se convirtió en un grito de protesta espontáneo del nacionalismo aplastado durante cuarenta años, pero fue reprimida brutalmente, no sólo por la policia sino también por las Boinas rojas, el cuerpo de paracaidistas. La indefensión de los estudiantes, frente a la maquinaria represiva de las fuerzas de seguridad, quedó demostrada por la violencia empleada. Fue algo más que una represión estudiantil. Fue una advertencia desesperada de las autoridades para que no volvieran a repetirse tales hechos. $Y$ sin embargo, los estudiantes regresaron a depositar velas encendidas alli donde habían sido golpeados. Ese 17 de noviembre puso en marcha la "Revolución de terciopelo" ${ }^{10}$.

Los miembros de la disidencia agrupados en la Carta 77 comprendieron que había llegado el momento de sacar al pueblo de la apatia y de establecer un programa de reivindicaciones junto a los estudiantes. En los teatros, en las facultades en los clubs se crearon foros de debate para analizar la situación y para atraer a muchos ciudadanos que, ese $17 \mathrm{de}$ noviembre se encontraban en sus casas de campo en las afueras de Praga. Ese era el precio que habían tenido que pagar por su silencio durante cuarenta años. Husák, que prefirió utilizar el término "consolidación" en vez de "normalización", había realizado un pacto tácito con la población. A cambio de calma política, había permitido que acumularan pequeños bienes de consumo, que practicaran una segunda economía para

Skvorecky, Josef: Talkin' Moscow Blues. London. Faber and Faber, 1989, pág. 241

Hrabal, Bohumil: Lettres à Doubenka. Paris. Laffont. 1991, pág. 118.

10 Garton ASH, Timothy: La chaudière. Europe centrale.1980-1990. Paris. Gallimard. 1990, pág. 380 . 
compensar la rigidez del sistema, creando una tela de araña de favores personales y de sobornos. El simbolo del pacto fue la casa de campo donde se refugiaban para olvidar la aceptación del régimen y acallar su culpabilidad "1".

Pero en la noche del viernes al sábado algo nuevo había acaecido en Praga. Como escribió el escritor Bohumil Hrabal: "Sucedió lo que tenía que suceder. El pueblo ha sido la fuerza determinante que ha barrido todo aquello que le impedia alcanzar la libertad ${ }^{12}$. Durante todo el fin de semana continuaron las manifestaciones. Los jóvenes salieron de nuevo a la calle y, junto a ellos, Alexandre Dubcek, el símbolo de la uPrimavera de Praga", satisfecho de haber visto el día en que los jóvenes, otros jóvenes distintos de los de hacía veinte años, le mostraban su agradecimiento. Pero Dubcek era el pasado, la renovación de la sociedad desde el Partido,el "socialismo con rostro humano" ${ }^{3}$.

Los estudiantes de Arte dramático decidieron convocar una huelga para el lunes 27 de noviembre e hicieron un llamamiento a otras Universidades para que ocupasen las facultades. En los teatros, los artistas lanzaron una declaración: «jNo espereis. Actuad!». Los directores y actores leyeron la convocatoria de los estudiantes al público que, emocionado, cantó el himno nacional. Por primera vez se produjo el encuentro entre la comunidad intelectual y la masa anónima de la población. El domingo 19, Havel tomó la dirección del movimiento y convocó a toda la oposición para formar un foro cívico llamado a convertirse en el portavoz del pueblo checoslovaco. La mayoria de los miembros del "Foro Cívico" eran intelectuales, antiguos disidentes, ex-miembros del Partido comunista, sacerdotes católicos y escritores del Pen Club. De un modo similar a la Carta 77, el "Foro Cívico" no surgió como un partido político, sino como una organización para aunar todas las tendencias de opinión, y como un medio para establecer un diálogo abierto con las autoridades comunistas sobre el futuro del pueblo checoslovaco.

La primera exigencia formulada por el «Foro Cívico» fue la dimisión de los dirigentes del Partido comunista comprometidos con la invasión de las fuerzas del Pacto de Varsovia en 1968, empezando por el Presidente Husák, el jefe del Partido comunista, Milos Jakeš, el Ministro federal del interior, Frantisek Kincl, y el Primer Secretario del Partido comunista de Praga,

WI: I IANS, Kieran: The Prague Spring and its aftermath.1969-1970. Cambridge. Cambridge University Press, pág. 40.

Hrabal. op. cit., pág. 146

Ibidem. 
Miroslav Štepán. Por otro lado, en Eslovaquia será otra organización, «Acción Pública contra la Violencia" quien tomará la dirección de la revolución, ante la tradicional desconfianza de los eslovacos contra la intelectualidad checa en la que veían un impedimento para sus aspiraciones nacionalistas.

La reacción inmediata del Partido comunista sólo podia aumentar la represión, pero ya era demasiado tarde. Las declaraciones de Gorvachov en la cumbre de Malta el 2 de diciembre abrieron una nueva era en el campo de las relaciones internacionales, en particular cuando afirmó que la Unión Soviética no intervendría en los problemas internos de Checoslovaquia, desaprobando formalmente, por lo demás, la invasión de 1968. A partir de ese momento se iniciaron las conversaciones entre el «Foro Civico" y el Primer Ministro federal, Ladislav Adamec que concluyeron cuando el 29 de noviembre la Asamblea federal votó eliminar de la Constitución el papel dirigente del Partido comunista. El 28 de diciembre Dubcek fue elegido Presidente del Parlamento que, por unanimidad, designó a Václav Havel Presidente de la República ${ }^{14}$.

La "Revolución de terciopelo" fue principalmente la revolución de los intelectuales. En Checoslovaquia los escritores siempre han encarnado la conciencia de la nación. denunciando todas las formas de opresión. Durante los cuarenta años de totalitarismo esa tradición adquirió una connotación particular. La palabra escrita se convirtió en el arma de los disidentes investidos de la misión social de despertar a la nación de su apatía. de su desmoralización. de su escepticismo ${ }^{\prime}$. Después del golpe comunista de 1948, fueron numerosos los escritores que recibieron con satisfacción el nuevo régimen. Milan Kundera explica su entusiasmo en aquella época por el hecho de pertenecer a un grupo de intelectuales llamados a cambiar el rumbo de la historia al término de la II Guerra mundial. como rechazo a los valores y a la politica de la democracia que abandonaron a Checoslovaquia en 1938 en manos de Hitler '. En las Universidades, los estudiantes comunistas emprendieron la tarea de reformar el sistema. Era. en palabras de Kundera, "la embraguiez del poder. la ocasión de poder saltar por encima de la historia trasformándola. e inaugurar una nueva época de la humanidad" ". Muchos jóvenes encontraron en la revolución del 48 un significado para sus inquietudes. una seguridad dentro del presente in-

Sobre el desarrollo de la "Revolucion de terciopelo. Existen numerosos Estudios. Una na racion dei dia a dia muy detallada en: Difapt in. Theodore: "A new nistory of the velvet revolution.. en The New rork Review. 17-12-1992

Havt L. op. cit. pag. $96-97$

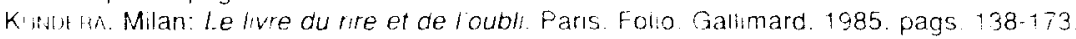
Kinllin M.: La broma. Barcelona. Selx. Barrai 1994 pag. 82. 
cierto de la adolescencia ${ }^{18}$. Pero pronto aquellos jóvenes que se sintieron el motor de la historia, como el mismo Kundera, se desengañaron del sueño socialista y abandonaron el Partido comunista. Se hicieron crítico. on su pasado y lucharon para salir de una historia que les había seducido ${ }^{1 y}$.

Bohumil Hrabal, que estuvo afiliado al Partido comunista durante nueve meses, permaneció en Checoslovaquia después de la invasión de las fuerzas del Pacto de Varsovia en agosto de 1968 al precio de "dejarme violar un poco para poder seguir viviendo aquí. Recibir el premio Klement Gottwald, la más alta distinción del Estado a un escritor. Aceptar la amargura, las bajezas, las entrevistas con los funcionarios del Ministerio del Interior para que mis libros fueran publicados" ${ }^{20}$. Pero para Hrabal, como para muchos otros escritores, permanecer en Checoslovaquia era la única manera de poder realizarse sin interrumpir el contacto con la lengua en continua evolución, aunque nunca le abandonó el sentimiento de culpabilidad ${ }^{21}$. Ése fue el secreto de su vida, tal vez el mismo que llevó a Sócrates a declarar antes de suicidarse "más vale obedecer a las leyes de la patria que emigrar» 22 .

La «Primavera de Praga» no había sido sólo obra de los políticos, sino también de los escritores. Quienes habian confiado en el cambio entraron en una semi clandestinidad. Ludvík Vaculík fue junto a Hrabal uno de los pocos que no emigraron después de la invasión. Vaculík creó la editorial "Pletice", donde los disidentes situados al márgen de la cultura oficial siguieron publicando en "samizdat", paradojicamente, dentro de una sorprendente libertad interior. La literatura "samizdat" fue un intento de cultura independiente realizada, inicialmente, por un pequeño círculo de escritores que se comprometieron a escribir sus manuscritos en máquina de escribir, haciendo varias copias en papel carbón para distribuirlas al márgen de las editoriales oficiales. Con el tiempo, el número de copias fue en aumento, del mismo modo que los trabajos y los lectores. La literatura "samizdat" se convirtió en el medio de expresión de la cultura reprimida por el régimen. Vaculík escribió que en una sociedad tan decepcionada como la checoslovaca, el papel del artista consiste en trasmitir la esperanza, aunque confiesa que no sabe muy bien a qué esperanza agarrarse. Sin embargo, demostró un gran realismo cuando afirmó en 1977

Kundera, M.: La vida está en otra parte. Barcelona. Seix Barral, 1989, pág. 223 Ibidem.

Hrafial op. cit.. pág. 179

Hatabal, Boumil: "La flúte enchantee", en Prague. Secrets et métamorphoses. Paris. Autrément. Mar, 1990, págs. 27-36.

SAl mon. Christian: A bâtons rompus avec Bohumil Hrabal. Paris. Criterion, 1991, pág. 49 
que el cambio político no sería logrado por los disidentes, sino por la nueva generación que no vivió los acontecimientos del 68 «el recien nacido sabe que todo es posible, todo puede sucederle e incluso puede triunfar». A pesar de la atmósfera axfisiante que le impuso el régimen de Husák, sabe que, "en la lucha para alcanzar la libertad, los primeros disidentes serán encarcelados. Los dirigentes no tendrán dificultades para encarcelar a diez o a mil más, pero perderán el poder cuando un millón de personas se opongan a ellos con obstinación» ${ }^{23}$.

La cultura oficial impuesta por los comunistas en 1948 significó la ruptura total de la nación con su pasado histórico. El arte, la literatura, la música, se convirtieron en un objetivo político ${ }^{24}$. El escritor, el pintor, el músico no podían reflejar sus sentimientos, sino sólo lo que expresase su solidaridad con el régimen. Uno de los personajes de Kundera, un conocido músico, critica el sistema que le hacia tocar en nombre de la lucha contra el imperialismo,o para conmemorar la revolución, o para celebrar el aniversario de un alto dirigente, obligado incluso a asistir a conferencias sobre el papel de la música en la edificación del socialismo ${ }^{25}$. El régimen comunista condenó a Checoslovaquia al olvido de su propia identidad. Kundera afirma que «la lucha del hombre contra el poder es la lucha de la memoria contra el olvido. La historia se evapora de la memoria y el escritor se ve obligado a hablar de hechos recientes como si ya tuviera mil años" ${ }^{26}$.

Después de la invasión de Checoslovaquia más de medio millón de checos abandonaron el país, y entre los que permanecieron, muchos intelectuales fueron obligados a cambiar su trabajo en fábricas oscuras, al volante de un camión o limpiando cristales, en aquellos lugares donde nadie escuchara jamás sus palabras. Kundera cuenta la historia de Tamina que se ve obligada a emigrar en el 68 sin poder llevar su diario. Poco a poco, su pasado se le aparece cada vez más difuso. Al principio intenta encontrar algunos recuerdos que le sirvan de punto de referencia en el devenir del tiempo, sus vacaciones de verano, las navidades. Pero cada vez le cuesta más encontrar el rítmo del tiempo. Ésa es la razón por la que Tamina intenta desesperadamente hacerse con su diario, para que el edificio tambaleante de sus recuerdos no se derrumbe y sólo le quede el presente que conduce inexorablemente a la muerte ${ }^{27}$.

23 Vaculik, Ludvík: La clef des songes. Paris. Actes-Sud, 1989, págs. 30-35 y 395.

24 RuPNIK, op. cit., pág. 271.

Kundera, M.: La valse aux adieux. Paris. Gallimard, 1986, pág. 33.

KunderA, M.: Le livre du rire et de l'oublie, op. cit., pág. 244.

Ibidem, págs. 138-173. 
Muchos años antes, Kafka había descrito Praga como una ciudad sin memoria, donde nadie se acuerda de nada. No es casual que las autoridades comunistas prohibieran sus libros en las Universidades, en las bibliotecas, en las librerias. Como explica el escritor Ivan Klima, la honestidad de Kafka no podía ser admitida por un régimen que se sustentaba de la decepción de los ciudadanos, a quienes pedía que expresaran externamente su adhesión sin tener en cuenta sus convicciones más profundas. La autenticidad de la obra de Kafka y su deseo de comprensión con sus semejantes le situaron junto a los disidentes contemporáneos ${ }^{28}$.

Contrariamente a lo esperado por Husák, la «normalización» supuso el nacimiento de una floreciente cultura underground que significó, no sólo el fin de la censura oficial, sino también el fin de la propia autocensura. La censura sólo permitía el discurso del régimen, en el que nadie creía y que probablemente tampoco importaba a los dirigentes. Pero la autocensura también habia tenido su efecto negativo, se pensaba una cosa en privado y se decía otra en público, y de este modo todos aceptaban el sistema contribuyendo a su mantenimiento, como dijo Václav Havel en su discurso de año nuevo en $1990{ }^{29}$. El propio Havel, que como portavoz de la Carta estuvo varias veces encarcelado, cuenta que antes de ser detenido por primera vez, se encontraba en un estado de angustia constante; no sabía qué debía escribir, con quién hablar, dónde ir, hasta que fue detenido. Entonces, frente a la pérdida de la libertad física, encontró su libertad moral ${ }^{30}$.

Los intelectuales checos se convirtieron una vez más en la conciencia de la nación. Mientras que en Polonia los "Samizdats" tuvieron una enorme difusión, en Checoslovaquia sólo repercutieron entre un círculo muy restringido. Pero esa minoría de escritores, de filósofos, de cantantes, de poetas, de comediantes se convirtió en un contra poder: "el poder de los sin poder ${ }^{31}$. En realidad los sin poder no luchaban por el poder porque ya lo tenian. Operaban fuera de las estructuras oficiales, y esa es la razón por la que el régimen les consideraba tan peligrosos, aunque sus únicas armas fueran sus ideas y sus palabras ${ }^{32}$.

Uno de los eslóganes de la «Revolución de terciopelo» fue «la verdad prevalecerá". Havel tomó del fundador de la República checoslovaca, To-

\footnotetext{
Kı. IMA. Ivan: Amour et ordures. Paris. Seuil, 1992. pág. 289.

La vie en rouge, op cit.. pág. 665.

HaVEL: op. cit. pág 169.

Havel, V.: El poder de los sin poder. Madrid. Ed. Encuentro. 1990. pág. 27.

RupNIK. op. cit.. pág. 309
} 
más Masaryk, el concepto moral del ciudadano y su comportamiento ético para asumir su responsabilidad. Masaryk no habia fundado un Estado como político realista, sino como pensador ${ }^{3.3}$. De un modo similar, la carta que Havel dirigió a Husák el 8 de abril de 1975 estuvo éticamente motivada, pero politicamente redactada. Por primera vez despues de la «Primavera de Praga", un intelectual tomó la palabra para contestar el omnipotente poder del Partido comunista. Havel denunció el miedo instaurado por el régimen como un miedo con sentido ético, y del que se derivaban la mentira y la corrupción moral ${ }^{34}$. Tanto Masaryk como Havel estuvieron preocupados por la base moral de la política y formaron parte de una oposición minoritaria que fue capaz de sustituir un régimen por otro en 1918 y en 1989, respectivamente. Ambos pasaron de la disidencia a la Presidencia de la República. En los dos casos, dos intelectuales se convirtieron en la encarnación de la nación y en los garantes morales de la democracia ${ }^{35}$.

Para Havel la política debe estar investida de una misión ética más allá de lo cultural o de lo espiritual. Ese sentido de la ética política, la responsabilidad, la honestidad para consigo mismo, fueron su único equipaje para llegar a la Presidencia. Havel realiza un constante análisis de su propia conciencia, y asume su culpabilidad como miembro de una sociedad que aceptó el régimen comunista durante cuarenta años, aunque fuera con resignación. Ese es el pecado de su existencia. Precisamente por ello, siente la necesidad de explicarse y defenderse continuamente ante los demás, para borrar su sentimiento casi metafísico de culpabilidad ${ }^{36}$. Es difícil entender cómo alguien sumido en un permanente análisis de su ética personal, sea al mismo tiempo capaz de desempeñar las labores de la Presidencia. El propio Havel responde que si se le considera un Presidente mejor que otro, es porque en su trabajo se esconde una duda constante sobre sí mismo y sobre el derecho que tiene a desempeñar tales funciones.

Václav Havel es conocido como un importante dramaturgo, un ensayista, y un disidente que por su oposición al régimen comunista en Checoslovaquia, y por la firmeza de sus convicciones tuvo que afrontar la cárcel en diferentes ocasiones. El nombre de Havel está asociado al teatro del absurdo pero, como señala Ivan Klíma, con anterioridad a la «Prima-

Casanova, Marina: “El problema de las minorias en Checoslovaquia durante la I República. 1918-1938", en Espacio. Tiempo y Forma. Madrid. UNED. 1995. pág. 113.

KLIMA. Ivan: The spirit of Prague. London. Granta Books, 1994. pag. 66.

GEL! NFA, Ernst: Encuentros con el nacionalismo. Madrid. Alianza Universal. 1994, pág.129 Have:., V.: Langoisse de la liberte. Eds. de l'Aube, 1994, págs. 7 y 92 
vera de Praga", cuando todavia se podian representar sus obras, el público las consideró sobre todo como obras políticas. Si Havel se convirtió en autor teatral fue sólo porque el teatro era la única plataforma donde podía expresar sus opiniones.. Para Klíma, Havel siempre ha sido, en primer lugar, un político, y luego un dramaturgo ${ }^{37}$. El mismo Havel, cuando escribe a su mujer Olga desde la cárcel en 1981, no considera que el teatro sea su destino, sino que ese medio le ofrece la posibilidad de ser él mismo: "precisamente yo, una persona esencialmente social y hasta diría política» ${ }^{38}$.

Cuando en 1989, en un breve espacio de tiempo, los sueños de los disidentes se hicieron realidad, Havel fue consciente de que la «Revolución de terciopelo" no habia convertido a Checoslovaquia en un oasis de paz, y de que, lo que todavía es más importante, cada ciudadano tenía que asumir la herencia de una conciencia moral deformada ${ }^{39}$. El discurso de Havel el 1 de enero de 1990 es una llamada a sus compatriotas para que asumiesen sus responsabilidades del pasado: «Vivimos en un medio moral y podrido. Estamos enfermos moralmente porque nos hemos acostumbrado a decir blanco y a pensar en negro. Hemos aprendido a no creer en nada, a no prestar atención al que tenemos al lado, a ocuparnos sólo de nosotros». El régimen comunista había convertido a los ciudadanos únicamente en medios de producción, en máquinas de no pensar, y Havel advierte duramente: «Todos nosotros nos hemos acostumbrado al sistema, lo hemos aceptado como algo inmutable. Todos nosotros, en mayor o en menor medida, somos responsables del funcionamiento de la máquina totalitaria. No somos sólamente sus víctimas, somos al mismo tiempo sus co-creadores" ${ }^{40}$.

Havel necesita compartir su culpabilidad y, al mismo tiempo, no se deja engañar por la realidad de un país que ha alcanzado la democracia. La herencia de cuarenta años de dictadura " no es un legado dejado por un pariente lejano, sino que hay que aceptarlo como algo que nosotros mismos hemos cometido contra nosotros. No podemos endosar la responsabilidad de todo a los gobiernos anteriores" ${ }^{4}$.

Este sentido de la responsabilidad de Havel, ha dado lugar a que algunos observadores consideren la transición en Checoslovaquia como un

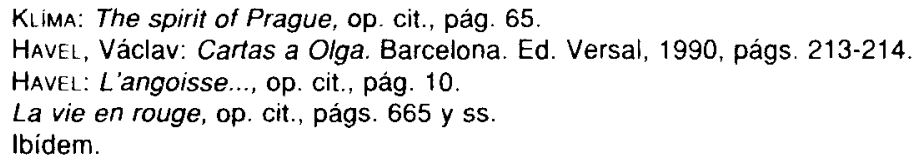


proceso de complicidad con el régimen anterior. Gellner ha señalado que todas las transiciones en Checoslovaquia contaron con la complicidad de vencedores y vencidos. Cuando los alemanes ocuparon el pais en 1939, el Presidente Hácha firmó un acuerdo con Hitler; el Comandante de las fuerzas alemanas negoció su retirada en 1945 con el Comité Nacional checo; el Presidente Beneš entregó el poder a los comunistas en 1948; Dubček firmó los Acuerdos de Moscú, y, finalmente, el Foro Cívico aceptó que un comunista dirigiera el primer gobierno democrático. Petr Pithart, firmante de la Carta 77, y Primer Ministro del gobierno checo en las elecciones de 1990, critica incluso el adjetivo "terciopelo" que califica la revolución, porque en su opinión ni siquiera hubo revolución, dado que no existió ningún conflicto para que las autoridades comunistas dejaran el poder ${ }^{42}$.

Las razones de este comportamiento en 1989, han sido justificadas por los nuevos dirigentes, entre ellos, por el hasta hace poco Primer Ministro, Václav Klaus, por la necesidad de reconvertir la economía del país con cierto pragmatismo, la introducción de una economía de mercado y el mantenimiento de algunos logros del sistema anterior. Por otro lado, a pesar de la Ley de responsabilidades politicas proclamada por el Parlamento en 1991, las actuaciones contra miembros de gobierno anteriores no han sido muy significativas ya que en opinión del Presidente Havel, es destructivo para el futuro continuar sumergidos en el pasado ${ }^{43}$.

Eda Kriseová, que trabajó junto a Havel, explica por qué los intelectuales no están hechos para ser políticos. Recuerda cómo en la Antigua Grecia muchos hombres que no eran profesionales de la política, fueron buenos políticos porque se sentían libres para tomar decisiones, porque en realidad no querían ser políticos. Para ellos, la moralidad debia ser el fundamento de todo político ${ }^{44}$. Así es como Havel aparece ante los ojos de Hrabal, como un héroe, que del mismo modo que los héroes de la Antigüedad, habia arrebatado el fuego sagrado a los comunistas. Por eso estuvo en la cárcel, y por eso, llegado el momento, se convirtió en la fuerza motriz de la "Revolución de Terciopelo" ${ }^{45}$. Con su amarga ironía, Hrabal considera que la revolución en Checoslovaquia se hizo a su manera: "Nuestro pueblo es gris como una paloma, gris como el amanecer, gris como la tormenta. Me digo a mí mismo: ¡qué bello pais!, y me pregunto

\footnotetext{
GellNER, op. cit., pág. 135.

Otfinoskı, Steven: The Czech Republic. New York. Facts on File, 1997, págs. 47-49

KaISEOvA, Eda: Václav Havel. El reto de la esperanza. Madrid. Espasa-Calpe. 1993, pág. 14.

SALMON, op. cit., pág. 53
} 
¿adónde va un país cuya revolución la han hecho posible los cómicos, los actores, los niños, los estudiantes?» ${ }^{46}$.

Ivan Klíma considera que la revolución de noviembre fue diferente a otras revoluciones, no sólo por la ausencia de violencia, sino por el arma empleada en la lucha: la ironia de la palabra. Todos los edificios públicos de Praga se cubrieron de carteles, de eslóganes, de pintadas. Los ciudadanos de Praga expulsaron al Partido comunista no con las armas, sino con la ironia ${ }^{47}$. Havel confiesa que los extranjeros nunca han entendido cómo el pueblo checoslovaco fue capaz de soportar cuarenta años de totalitarismo, y al mismo tiempo no dejar de ironizar, y añade que, en Praga, en Bohemia y en Europa central, los acontecimientos dolorosos siempre se han mezclado con el sentido de la ironía, del ridículo o del humor negro ${ }^{48}$.

En Checoslovaquia, después de la "Revolución de terciopelo" gobernaron los intelectuales porque no había políticos. Los disidentes estaban acostumbrados a decir la verdad, eran autocríticos con el poder, pero no sabían cómo poner en práctica sus ideas. Desde 1990 hasta 1992 vencieron los intelectuales, pero fue el pragmatismo de Václav Klaus quien que ganó las elecciones de 1992. Si en política algunos piensan que el intelectual siempre estará vencido de ante mano y condenado a perder, para Havel, el intelectual nunca será vencido porque ganará perdiendo ${ }^{49}$. 